\title{
Management of chronic pain
}

\author{
Stephen P. Tyrer
}

Until 50 years ago chronic pain was not considered to be a medical condition that required special evaluation and treatment facilities. Pain was considered to be an indicator of tissue damage and appropriate medical or surgical treatment was prescribed for this. The many patients who had continuing pain because of the results of disease or trauma or because acute treatment was unable to relieve the condition were not seen as suffering from a recognised pathological entity. It was not until a doctor, Sicilian-born John J. Bonica, had to pay his debts as a medical student through wrestling professionally, and later suffered persistent pain as a result, that chronic pain became a recognised condition in its own right. Bonica started a pain clinic in Tacoma, Washington State, in 1949 and wrote the first textbook on pain treatment, The Management of Pain, in 1953. There are now 2000-3000 pain clinics in the USA and almost 5000 worldwide.

It was recognised early that no single specialist could address all the complexities presented by these patients, and Bonica employed physicians and psychologists in his first pain clinic. The multidisciplinary pain clinic is now considered the ideal facility for the treatment of pain. There are now some 250 pain clinics in the UK but the majority of these are run by anaesthetists with little input from other health care professionals.

Although this article is primarily concerned with the psychological and psychiatric aspects of the management of chronic pain, a brief account will be given of techniques used by others in this condition.

\section{Pain management clinics}

A pain management clinic may offer in-patient and out-patient facilities but the vast majority in Britain are for out-patients only. In-patient programmes may be required for those severely ill with pain from cancer, for patients undergoing a procedure that requires post-operative monitoring, if there is a major problem with medication abuse, and for those individuals who require a behaviour-based programme because of the severity, chronicity and psychosocial consequences of their pain.

All patients attending any pain management clinic first require an accurate evaluation of the degree and extent of organic pathology (current physical symptoms and past evidence of tissue damage). A few patients may be referred on to another specialist because of acute pathology or if they have a condition which is amenable to specific treatment (e.g. surgery) but the majority of patients can be treated in the clinic.

The organisation and staffing of such clinics depends upon what treatment services are offered, but there are many advantages in having such clinics on a general hospital site (Charlton, 1992).

Although many patients attending pain clinics have existing medical conditions (e.g. hypertension, diabetes) it is appropriate for the painful component of their medical condition to be treated by pain clinic specialists as long as there is no progressive pathology and no medical or surgical intervention indicated. Furthermore, if the patient believes that a surgical or medical technique is likely to be of benefit, he or she will be less inclined to cooperate with cognitive and behavioural procedures in the pain clinic. It is quite common for patients to have unrealistic expectations of specific medical or surgical treatments and these misconceptions need to be addressed before deciding on appropriate treatment strategies.

\section{Diagnoses of patients}

The majority of patients attending pain clinics have had evidence of a physical cause for pain acquired through injury or disease. However, in many

Stephen Tyrer is a Consultant in Pain Management and Psychiatry at the Pain Management Unit, Royal Victoria Infirmary, Newcastle upon Tyne NE1 4LP. He has worked in the chronic pain field for 15 years and has been Visiting Professor at the Chedoke Rehabilitation Program for Chronic Pain at McMaster University, Hamilton, Ontario, Canada. 
patients there is no evidence for continuing pathology, at least based on unequivocal signs on examination, X-ray findings, MRI scans or biochemical and/or haematological indices of illness. Box 1 lists the most common syndromes suffered by attenders at UK pain clinics (see Tyrer et al, 1989).

\section{Psychological and psychiatric factors}

Early on it was recognised that psychological factors were of prime importance in the evaluation of pain. The Taxonomy Committee of the International Association for the Study of Pain (Merskey, 1979) stated that:

Activity induced in the nociceptor and nociceptor pathways by a noxious stimulus is not pain, which is always a psychological state ...

The nociceptor refers to the sensory nerve end, stimulation of which gives rise to the characteristic sensation of pain. Physical and psychological assessment is needed for all patients attending the pain clinic, although in the absence of experts screening instruments can be used to determine whether particular psychological treatment strategies are necessary.

It is customary and sensible for the physical state of the patient to be assessed first on referral to the pain clinic, before the psychologist or psychiatrist sees the patient. There are advantages if the physician assessing the psychological and psychiatric contributions to the chronic pain is also experienced in the assessment of physical factors that cause pain, allowing the influence of overshadowing physical factors to be evaluated accurately (Tyrer, 1992a). An accurate account

Box 1. Diagnoses of patients attending pain clinics in the UK

Neuralgias/neuritides

Low back pain

Myofascial pain syndromes

Joint disease, mainly osteoarthritis

Headache

Facial pain

Sympathetically mediated pain, e.g. complex regional pain syndromes

Cancer

Vascular damage

Trauma

Multiple sclerosis should be obtained of the circumstances surrounding the onset of the pain. If there is exceptional mental or physical stress just before the painful event, a post-traumatic stress disorder or adjustment disorder may accompany the painful symptoms and contribute to their maintenance.

Enquiry should be made about the intensity of the pain. Most patients are able to rate their past and present pains on a 10-point Visual Analogue Scale (Seymour et al, 1985). When patients are in distress from physical or psychiatric factors they tend to rate the intensity of their pain as higher than when they are in a euthymic state (Price et al, 1995). Those who are convinced that they have a definite illness and who are disinclined to accept help have longer-standing pain and greater psychiatric morbidity (Elton et al, 1994).

Pain that occurs in multiple sites in the body, that has increased in area over time, that is described as "dreadful" or "punishing", that does not wake the patient from sleep or that is unaffected by movements, is more likely to be found in patients who have some contribution from psychological and psychiatric factors. Examination signs that are associated with non-organic pathology include: over-reaction to examination, superficial tenderness, expression of pain when manoeuvres are employed which the patient believes will cause pain but in fact do not affect bodily mechanics (e.g. axial loading through pressure on the head) and variable performance when distracting tests are used (e.g. ability to sit comfortably with legs outstretched but not be able to extend the legs fully when lying supine) (Waddell et al, 1980).

The psychiatric factors involved in chronic pain are illustrated in Table 1. Concern about the cause of illness, disease conviction and degree of illness behaviour are more salient in the assessment than psychiatric diagnosis. Although the majority of patients seen in a pain clinic have a measurable degree of psychological distress, less than one-third have a psychiatric illness, the most common of which is depression (Pilowsky \& Bassett, 1982; Tyrer et al, 1989). It has been proposed that depression increases the sensory transmission of pain by facilitating pain pathways along afferent neurones as well as opening the 'gate' in the dorsal horn of the spinal cord. As depressed individuals are more likely to interpret life events as being threatening, this affects both the evaluative and affective aspects of the pain experience (Fields, 1991). There is some experimental evidence to support this theory (Geisser et al, 1994).

The fact that some patients become incapacitated by apparently trivial painful complaints has intrigued their doctors since the time of Breuer and Freud. Engel (1959) encapsulated many of these concepts when he described a group of pain-prone 
patients in whom there was a background of parental abuse, sometimes followed by remorse and compensating affection. In this way the child who does not receive much affection at other times, becomes accustomed to the sequence of pain and suffering being followed by love.

Some patients express their affect and anger by complaining of pain or other somatic mechanisms (Sifneos, 1973), whereas others develop 'Athlete's neurosis', when injury or illness impairs their activities (Little, 1969).

\section{Management strategies}

Optimal treatment should be provided for any patient in pain who has a clear organic aetiology (e.g. non-steroidal anti-inflammatory agents in osteoarthritis, sympathetic block in complex regional pain syndromes). However, in many cases it is appropriate to provide symptomatic relief irrespective of the origin of the pain and the following strategies (see Box 2) may be considered in approximate order of priority.

\section{Transcutaneous electrical nerve stimulation (TENS)}

The use of electrical nerve stimulation to reduce pain was known to Hippocrates around $40 \mathrm{BC}$, who used the electric torpedo fish for the treatment of headache and arthritis. However, it was not until Melzack \& Wall (1965) proposed the 'gate' control theory of pain
Box 2. Physical treatments in chronic pain

Transcutaneous electrical nerve stimulation Anaesthetic nerve blocks

Analgesic drugs

Acupuncture

Physiotherapy

Occupational therapy

that attempts were made to reduce pain by the stimulation of low-threshold touch fibres at the level of the dermatome where pain afferent impulses were impinging on the dorsal horn of the spinal cord. Portable battery-operated stimulators for pain relief have been available since 1973, which block transmission of pain impulses in the dorsal horn of the spinal cord. TENS is of most value in somatic pains and pains arising from nerve damage and muscle tension; it is of least advantage in pain for which no organic cause is apparent.

If a physician believes that TENS is appropriate, a trial is normally carried out with the instrument, with the electrodes applied over the site of pain. It can be used throughout the waking hours. It has the advantages or being patient-controlled, there are very few adverse effects and virtually no contraindications to its use. Relative contraindications are that it should not be used over the anterior part of the neck because of the danger of stimulating the carotid sinus, or over the pregnant uterus. An absolute contraindication is that it cannot be used in the presence of a cardiac pacemaker. TENS is now available in continuous

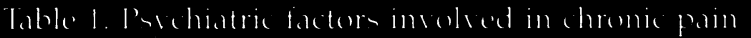

Cause or mental mechanism Significance in patients with chronic pain

Anxiety

Depression

Hysteria

Hypochondriasis

Patients with somatisation disorder display a combination of hypochondriacal and hysterical symptoms and seek medical help for a wide variety of symptoms

Drug dependence

More common in patients given opioid drugs. More likely in patients with personality disorder; particularly passive-dependent personalities

Psychosis

Increased anxiety is associated with increased pain; may be linked with muscle tension

Most common psychiatric symptom in patients with chronic pain with depression usually resulting from the pain

Often no more than unconscious increase in existing symptoms for a variety of motives, although there are isolated cases where pain provides a solution for an unconscious conflict

The element of disease conviction is very common, particularly in patients with no demonstrable evidence of physical illness. Fear about disease is uncommon

Rare but definite cases of pain can arise in schizophrenia and psychotic depression, occasionally in monosymptomatic hypochondriacal psychosis 
or pulse mode and there is evidence that the pulse or burst mode is more effective in most patients.

\section{Diagnostic and therapeutic anaesthetic nerve blocks}

Nerve blocks are widely used in pain clinics in Britain. This is largely because most pain clinics are run by anaesthetists who have expertise in this measure. The dramatic effect of blocking transmission in afferent pain nerves also has an iatrogenic benefit in itself.

Where it is suspected that peripheral nerve damage is giving rise to pain it is normally appropriate to carry out a diagnostic nerve block. The purposes of diagnostic blockade include diagnosing the site of pain, identifying any contribution from the sympathetic nervous system, separating local disease from referred pain and determining whether a neurogenic pain disorder arises from central or peripheral mechanisms.

If a diagnostic block improves pain and function in the blocked area, the time course of the response is appropriate to the agent employed and there is objective evidence of the action of the block with appropriate sympathetic, sensory or motor changes, therapeutic blockade may be of benefit. However, the belief that therapeutic nerve block is an advantage in chronic pain is coming under increasing scrutiny as the momentum grows for the implementation of evidence-based medicine. There is little hard evidence from well-constructed clinical trials that favour the use of therapeutic nerve block as a method of treatment. The best examples have been found in neurolytic blockade for pain arising from cancer (Boys et al, 1993). However, there is always the danger in any destructive or permanent procedure involving damage to the nerves that pain may return because of damage to the existent efferent stump. Where nerve destruction cannot be contemplated there is anecdotal evidence that serial blockade with long-acting local anaesthetic agents such as bupivacaine can benefit a wide variety of conditions (Stolker et al, 1994).

If there is benefit, the reduction in pain that follows blockade should be accompanied by aggressive physiotherapy to aid return to normal function.

\section{Drugs for pain}

There are five main groups of drugs used in the treatment of pain (see Box 3): non-acidic antipyretic analgesics (e.g. paracetamol) and non-steroidal antiinflammatory drugs (NSAIDs) act largely peripherally, whereas opioid drugs, antidepressants and anticonvulsants act largely centrally.
Box 3. Use of drugs in chronic pain

Simple analgesics (e.g. paracetamol, coproxamol) - effective for less severe pains; safe in low dosage; non-addictive

Non-steroidal anti-inflammatory drugs useful only if there is evidence of inflammation; risk of epigastric pain and gastrointestinal irritation

Opioids - more useful in central pain conditions; few problems of dependence

Antidepressants - analgesic in low dosage

Anticonvulsants - may be effective in pains due to nerve damage (e.g. neuralgias, phantom limb pains)

By the time a chronic painful state has emerged, the standard conventional drugs that are used in the treatment of pain are usually of little benefit. Paracetamol is the safest agent to use and can be given at a dosage of up to eight tablets $(4 \mathrm{~g})$ every 24 hours. Aspirin and the NSAIDs are rarely of value unless there is evidence of a chronic or an acute-onchronic inflammatory process. Opioids can be given to most patients with chronic non-malignant pain without dependence supervening. However, many find that side-effects of somnolence, reduced energy and constipation are limiting factors. Patients with a pre-existing dependent personality disorder or antisocial personality disorder are more likely to misuse these drugs.

In contrast, the antidepressant and anticonvulsant drugs do not usually cause untoward side-effects in the low doses at which they are effective in chronic pain. Anticonvulsants certainly reduce the transmission in afferent pain nerves and it seems likely that antidepressants in low doses act in the same way (Magni, 1991). In theory, they are more likely to be effective in patients who have pure neurogenic pains (e.g. post-herpetic neuralgia) although they can improve pains arising from structures where there is no evidence of nerve damage (e.g. atypical facial pain; Feinmann et al, 1984).

\section{Drugs for psychiatric illness}

Although depression and, to a lesser extent, anxiety are common features in patients with chronic pain, the symptom profile of patients in chronic pain is manifestly different from those with endogenous depression (Pilowsky \& Bassett, 1982). 
Antidepressants are of benefit in treating depressive symptoms but have less effect on the intensity of pain itself (Ward, 1986).

\section{Acupuncture}

Acupuncture is another example of peripheral stimulation-induced analgesia that works in a different way to TENS. The efficacy of acupuncture is best for myofascial and musculo-skeletal pains (Gaw et al, 1975). Although there is some evidence that acupuncture carried out at traditional Chinese acupuncture points is associated with endorphin release, meta-analysis of the literature reveals highly contradictory results (Ter Riet et al, 1990).

\section{Physiotherapy}

Physiotherapy is of the most use within the first few months of tissue damage but may be of value in those who have adopted abnormal postures or in those who have barely exercised since the onset of the condition causing their pain. Assessment by the physiotherapist includes determination of muscle strength, limitation of joint movements, pattern of gait, posture, degree of muscle tension, cardiovascular endurance and body mechanics. After the assessment period an individual programme designed to improve function is devised and is particularly directed towards activities that the patient would like to restart but has given up because of pain. Homework exercises are encouraged and gradually increased if there is improvement. The basic goal of any pain clinic is restoration of function and physiotherapy is an integral part of that process.

\section{Occupational therapy}

Occupational therapy normally should be carried out after the patient has made some gains with physiotherapy and other treatments. The aim is to use those gains to improve daily activities, including self-care, home management, leisure and occupational pursuits.

\section{Psychological treatments}

\section{Behavioural therapies}

Sufferers from chronic pain adopt a variety of strategies which are not always of benefit in the long term. In particular, if a patient believes that he or she has a clear physical illness because of continuing pain, illness behaviour can result which has the potential to become persistent if there are advantageous results. Fordyce recognised early on that many patients were apprehensive and reluctant to maintain their former activities because of the worry that this would increase pain. In some cases there were positive advantages in the patient adopting the 'sick role' (e.g. cessation of an unpleasant manual occupation, solicitous help from a previously inattentive spouse; Tyrer, 1986). Fordyce et al (1973) developed behavioural programmes based largely on the principles of operant conditioning in which both physical and psychological gains in treatment are rewarded appropriately.

The treatments proposed by Fordyce are based on the assumption that pain is a handicap only if it leads to behavioural impairments. If the patient is able and willing to carry out both vocational and occupational activities despite the pain and can do this successfully, there will be improvement in function and selfesteem which enable the patient to cope successfully. Patients who complete such programmes usually find that although their pain is not greatly reduced, they are better able to manage their pain, in part because of the cognitive benefits resulting from the physical and psychological gains that they have achieved (Turner, 1988). How far this improvement is due to operant factors or to what extent erroneous cognitions have been altered is a moot point.

\section{Cognitive therapies}

Patients in chronic pain have thoughts and beliefs about their pain which are not always justifiable. For instance, a common assumption is that because pain interferes with a desired activity, the activity concerned should no longer be carried out under any circumstances. It may be possible to modify an activity that was previously perceived as pleasurable by the patient before the onset of chronic pain, and which can be practised again with modifications to account for the handicaps that have resulted from the painful condition. The process concerned is similar to other forms of cognitive treatment. There needs to be an assessment of the type and intensity of faulty cognitions, the extent of depression and the incidence of maladaptive behaviour patterns (e.g. excessive guarding).

First it is necessary to find out the patient's beliefs about their pain or 'illness representation'. Suggested lead questions by the therapist include: "What ideas do you have about the cause of your pain?"; "What are your expectations with regard to treatment?". There may be erroneous ideas about how the pain has been caused and how it 
has affected the patient's functioning. Patients commonly are apprehensive about carrying out activities because they believe they will cause increased damage to tissues, although in practice inactivity is a greater problem. Matters are more obscure because the pain therapist often cannot fully explain the origin of the patient's pain on pathological grounds. It is vital that an attempt is made to convey to the patient why they are in pain using the knowledge that has been gained about the principles of pain perception.

It is also important to recognise that patients in chronic pain are most unlikely to be 'cured' of their pain; indeed, all the evidence suggests that the degree of pain does not alter a great deal, whatever the nature of the treatment proposed. However, patients can become better able to manage their pain and have a greater understanding of what factors affect their degree of pain.

Once dysfunctional cognitions have been identified it is important to note these 'automatic beliefs' and determine what events lead to them and their consequences. The patient keeps a diary of events and thoughts and the therapist then collaborates with the patient to challenge any ideas that are recognised to be faulty. The events leading to the beliefs and the consequences of engaging in the automatic thoughts should be recorded. Alternative strategies should then be selected to challenge these.

Thus, a man with recurrent chest pain following a cardiac by-pass for which there is no cardiovascular cause, may believe every time that he gets chest pain that he is going to have another heart attack. If it can be demonstrated to him that there is no likelihood of this occurring (here it is often helpful to show the patient the results of tests such as exercise ECGs), the patient can be encouraged slowly to increase activities and pause when he develops chest pain but not give up the activity altogether.

It should be recognised that the focus of these therapies is not to solve a particular problem but to manage emotions that disrupt healing strategies. The aim is not to eliminate all negative emotions but to decrease their intensity when they truly result from negative distortions of events and situations. Cognitive coping strategies that are used specifically in chronic pain include: increasing tolerance to pain, mood management, positive thinking, imagery, attentional diversion, and increasing self-control of pain. An effective instrument to determine these is the Coping Strategies Questionnaire (Rosenstiel \& Keefe, 1983).

Increased tolerance to pain accompanies the recognition that the presence of pain does not mean that there is continuing physical damage. Efforts should be made to minimise the sufferer's disability and use cognitive distraction and relaxation techniques. Stress management techniques include encouragement of sleep routines, physical exercises, avoiding procrastination and 'pacing' (carrying out an activity in short stages and pausing from it when symptoms are identified which are known to lead to adverse consequences, e.g. increased pain, stiffness).

Diverting attention away from pain by means of imagery or other techniques is often of benefit. Imagery can be used in a number of ways including imaginative inattention (e.g. lying in the sun), transformation of pain into numbness or tingling, and transformation of the context of pain (e.g. imagining that this is as a result of running a longdistance race). Attentional diversion uses techniques to attend to other distractors than pain including focusing on the immediate environment (e.g. colours, shapes, texture), concentrating on mental activities or plans and paying attention to alternative bodily sensation such as warmth, sensations when breathing and feelings of touch.

Once the patient recognises that their own activities are of more benefit in helping them manage their pain themselves, with progression from a patient to a non-patient status, a major change has occurred. This change can be helped by the therapist questioning further the sufferer about factors underlying the pain and encouraging the patient to try any strategy which he or she thinks may be helpful in reducing the pain.

It is important to have a procedure for dealing with times when the patient's pain has become worse, whether for physical or emotional reasons. It is acceptable to discontinue a programme of rehabilitation for one or two days if there are good reasons, which must be identified beforehand in conjunction with all concerned.

The value of cognitive therapy in reducing pain behaviours has been shown in a number of studies (Turner, 1988; Turk \& Meichenbaum, 1994).

\section{Relaxation and hypnosis}

Most patients with chronic pain can benefit from relaxation procedures. The conventional wisdom is that it is necessary to induce relaxation in the hospital or office setting and then reinforce any gains using a relaxation tape and techniques to decrease distraction. Bio-feedback may be of benefit (Cott et al, 1992). Once relaxation has been achieved, relaxation responses (i.e. the patient saying a word or breathing deeply with an underlying cue word) may help considerably in enabling the patient to avoid painful situations during the day. 
Box 4. Psychological treatments in chronic pain

Behavioural therapy - particularly in patients who have considerably reduced activity

Cognitive therapy - particularly in those with dysfunctional beliefs about their pain and who have few coping strategies

Relaxation - valuable in those with muscle tension and anxiety

Hypnosis is often a sought-after option by the patients although the main aim of this technique should be to encourage self-relaxation procedures (Tyrer, 1992b).

Psychological treatments for chronic pain are summarised in Box 4.

\section{Vocational assessment and counselling}

If sufficient improvement is shown by these techniques and the patient wishes to return to work in a part-time or full-time capacity, the Disablement Employment Advisor, Employment Medical Advisor, or local rehabilitation unit should be contacted. There are many advantages in part-time work in the first instance.

\section{Conclusions}

The management of chronic pain requires a good knowledge of pathology, behavioural psychology and rehabilitation techniques. Although success is often not great when measured by reduction in intensity of pain, considerable gains can be achieved in enabling patients to manage their pain better without relying on health care professionals. The benefits in this field come from increasing the self-knowledge and self-reliance of the pain sufferer, rather than in achieving a 'cure'.

\section{Acknowledgement}

Thanks to Dr Ed Charlton of the Pain Management Unit in Newcastle for helpful comments and advice.

\section{References}

Bonica, J. J. (1953) The Management of Pain. Philadelphia, PA: Lea \& Febiger.

- (1986) Past and current status of pain research and therapy. Seminars in Anaesthesia, 5, 82-99.

Boys, L., Peat, S. J., Hanna, M. H., et al (1993) Audit of neural blockade for palliative care patients in an acute unit. Palliative Medicine, 7, 205-211.

Charlton, J. E. (1992) Organisation of services in the pain clinic. In Psychology, Psychiatry and Chronic Pain (ed. S. P. Tyrer), pp. 189-200. Oxford: Butterworth Heinemann.

Cott, A., Parkinson, W., Fabich, M., et al (1992) Long-term efficacy of combined relaxation biofeedback treatments for chronic headache. Pain, 51, 49-56.

Elton, N. H., Magdi, M. H. \& Treasure, J. (1994) Coping with chronic pain. Some patients suffer more. British Journal of Psychiatry, 165, 802-807.

Engel, G. (1959) 'Psychogenic' pain and the pain-prone patient. American Journal of Medicine, 26, 899-918.

Feinmann, C., Harris, M. \& Cawley, R. (1984) Psychogenic pain: presentation and treatment. British Medical Journal, 228, 436-438.

Fields, H. (1991) Depression and pain: A neurobiological model. Neuropsychiatry, Neuropsychology and Behavioural Neurology, 4, 83-92.

Fordyce, W., Fowler, R., Lehmann, J., et al (1973) Operant conditioning in the treatment of chronic clinical pain. Archives of Physical Medicine and Rehabilitation, 54, 399-408.

Gaw, A. C., Chang, L. W. \& Shaw, L.-C. (1975) Efficacy of acupuncture on osteoarthritic pain. New England Journal of Medicine, 293, 375-378.

Geisser, M. E., Robinson, M. E., Keefe, F. J., et al (1994) Catastrophizing, depression and the sensory, affective and evaluative aspects of chronic pain. Pain, 59, 79-83.

Little, J. C. (1969) The athlete's neurosis - a deprivation crisis. Acta Psychiatrica Scandinavica, 45, 187-197.

Magni, G. (1991) The use of antidepressants in the treatment of chronic pain: A review of the current evidence. Drugs, 42, 730-748.

Melzack, R. \& Wall, P. W. (1965) Pain mechanisms: a new theory. Science, 50, 971-979.

Merskey, H.(1979) Pain terms; a list with definitions and notes on the usage. Recommended by the IASP Sub-Committee on Taxonomy. Pain, 6, 249-252.

Pilowsky, I. \& Bassett, D. L. (1982) Pain and depression. British Journal of Psychiatry, 141, 30-36.

Price, D. D., Gracely, R. H. \& Bennett, G. J. (1995) The challenge and the problem of placebo in assessment of sympathetically maintained pain. In Reflex Sympathetic Dystrophy; $A R e$ Appraisal (eds W. Jannig \& M. Stanton-Hicks). Progress in Pain Research and Management, vol. 6. Seattle, WA: IASP Press.

Rosenstiel, A. K. \& Keefe, F. J. (1983) The use of coping strategies in chronic low back pain patients. Relationship to patient characteristics and current adjustment. Pain, 17, 33-44.

Seymour, R. A., Simpson, J. M. \& Charlton, J. E. (1985) An evaluation of length and end-phrase of visual analogue scales in dental pain. Pain, 21, 177-185.

Sifneos, P. (1973) The prevalence of 'Alexithymic' characteristics in psychosomatic patients. Psychotherapy and Psychosomatics, 22, 255-262.

Stolker, R. J., Vervest, A. C. M. \& Groen, G. J. (1994) Management of chronic spinal pain by blockades: A review. Pain, 58, 1-20.

Ter Riet, G., Kleijnen, J. \& Knipschild, P. (1990) Acupuncture and chronic pain: A criteria-based meta-analysis. Journal of Clinical Epidemiology, 43, 1191-1199.

Turk, D. C. \& Meichenbaum, D. (1994) A cognitive-behavioral approach to pain management. In Textbook of Pain (3rd edn) (eds P. D. Wall \& R. Melzack), pp. 1337-1348. Edinburgh: Churchill Livingstone.

Turner, J. A. (1988) Comparison of operant behavioral and cognitive behavioral group treatment for chronic low back pain. Journal of Consulting Clinical Psychology, 56, 261-266. 
Tyrer, S. P. (1986) Learned pain behaviour. British Medical Journal, 292, 1-2.

- (1992a) Psychiatric assessment of chronic pain. British Journal of Psychiatry, 160, 733-741.

- (1992b) Hypnosis. In Psychology, Psychiatry and Chronic Pain (ed.S. P. Tyrer), pp. 149-155. Oxford: Butterworth Heinemann.

-, Capon, M., Peterson, D. M. et al (1989) The detection of psychiatric illness and psychological handicaps in a British pain clinic population. Pain, 36, 63-74.

Waddell, G., McCulloch, J., Kummel, E., et al (1980) Non-organic physical signs in low back pain. Spine, 5, 117-125.

Ward, N. G. (1986) Tricyclic antidepressants for chronic low back pain: Mechanism of action and predictors of response. Spine, 11, 661-665.

\section{Multiple choice questions}

1. Patients with chronic pain:

a are usually convinced they have a disease

b have been shown to have evidence of increased nerve conduction up afferent nerves

c complain of more pain when depressed

d often have no evidence of any physical pathology

e have a depressive illness in $10-15 \%$ of cases.

2. Drugs that are effective in chronic pain include:

a sodium valproate

b haloperidol

c naltrexone

d dothiepin

e diclofenac.
3. The following cognitive coping strategies have been found to be effective:

a focusing on the cause of the pain

b imagery

c attentional diversion

d replacing the painful sensation with one of warmth

e stimulation-induced analgesia.

$\begin{array}{cll}\text { MCQ answers } & & \\ \text { 1 } & \text { 2 } & 3 \\ \text { a T } & \text { a T } & \text { a F } \\ \text { b F } & \text { b F } & \text { b T } \\ \text { c T } & \text { c F } & \text { c T } \\ \text { d F } & \text { d T } & \text { d T } \\ \text { e T } & \text { e T } & \text { e F }\end{array}$

